\title{
Temporal evolution of longitudinal bunch profile in a laser wakefield accelerator
}

\author{
M. Heigoldt, A. Popp, K. Khrennikov, J. Wenz, S. W. Chou, and S. Karsch ${ }^{*}$ \\ Max-Planck-Institut für Quantenoptik, Hans-Kopfermann-Strasse 1, 85748 Garching, Germany \\ and Ludwig-Maximilians-Universität München, Am Coulombwall 1, 85748 Garching, Germany
}

\author{
S. I. Bajlekov and S. M. Hooker \\ Department of Physics, University of Oxford, Clarendon Laboratory, Parks Road, \\ Oxford OX1 3PU, United Kingdom \\ B. Schmidt
}

Deutsches Elektronen-Synchrotron DESY, Notkestrasse 85, 22607 Hamburg, Germany (Received 4 August 2014; revised manuscript received 17 June 2015; published 16 December 2015)

\begin{abstract}
We present single-shot measurements of the longitudinal bunch profile from a laser-wakefield accelerator with sub-fs resolution, based on detection of coherent transition radiation in a broad spectral range. A previously developed phase retrieval algorithm enables reconstruction of the bunch profile without prior assumptions about its shape. In this study, a variable-length gas target is used to explore the dynamics of bunch evolution. Our results show that once the laser energy is depleted the time structure of the generated electron beam changes from a single bunch to a double bunch structure, well suited for driverwitness type experiments.
\end{abstract}

Since the first demonstration of high-quality, quasimonochromatic electron beams in 2004, laser wakefield acceleration (LWFA) has become a reliable scheme to accelerate electron bunches to energies in the $\mathrm{GeV}$ range in plasma accelerator stages a few cm long [1-5]. The small extent of the acceleration structure that confines the bunch to a fraction of the plasma wavelength implies bunch durations in the few-femtosecond range. Experimental access to the detailed form of the longitudinal electron bunch profile, and its evolution with propagation through the accelerator, is crucial for a deeper understanding of the physical mechanisms involved in wakefield accelerators. It also provides an important diagnostic for monitoring and optimizing LWFA electron bunches in future applications, such as compact sources of ultrashort X-ray pulses, e.g., all-optical FELs, Thomson-scattering or betatron sources [6-11]. Recent experiments confirm the ultrashort nature of LWFA electron beams [12,13] and the acceleration of discretely spaced bunch trains [14]. However, to date no experiments have been able to provide detailed information about the bunch shape or its evolution within the accelerator. Multibunch structures are of particular interest for

\footnotetext{
*Corresponding author. stefan.karsch@mpq.mpg.de

Published by the American Physical Society under the terms of the Creative Commons Attribution 3.0 License. Further distribution of this work must maintain attribution to the author $(s)$ and the published article's title, journal citation, and DOI.
}

plasma wakefield acceleration (PWFA), in which a highgradient wakefield is driven by a dense particle bunch to provide an accelerating wakefield for a subsequent witness bunch. This scheme has the benefit of avoiding the relative phase slippage between the wake driver and the accelerated bunch that is inherent to LWFA and ultimately limits the achievable energy gain per stage [15]. While PWFA has been studied both theoretically and experimentally [16-19], the production of a well-controlled dense driver and a synchronized witness bunch is a demanding task, even for current state-of-the-art linear accelerators. As we show in this work, under certain conditions, such a double bunch structure may be conveniently produced in a single LWFA stage.

Likewise $[13,14]$ our bunch profile diagnostic is based on a frequency-domain technique by measurement of the intensity spectrum of coherent transition radiation (CTR). Compared to earlier work, a key improvement is the single-shot coverage of a broadband spectral range of more than 4 octaves, extending from visible to midinfrared frequencies, which ultimately yields a time resolution of the reconstructed bunch profile in the sub-fs region. Moreover, the CTR spectrum was analyzed with a new algorithm [20] which does not assume a form for the longitudinal bunch profile or extrapolation of the spectrum outside the measured range. Utilizing the remarkable shot-to-shot stability of the electron beam obtained by using a turbulence-free steady-state flow gas cell and a well-controlled laser system, we were able to produce electron beams with low fluctuations in spectral 
shape, charge and cutoff-energy. Together with the singleshot reconstruction technique, a scan of the interaction length allows us to observe the plasma dynamics during the acceleration process.

CTR is produced by the passage of a bunch of charged particles through the boundary between media with different dielectric indices. For a cylindrically symmetric bunch of $N_{e}$ electrons, the emitted energy $W$ at frequency $\omega$ in observation direction $\theta$ is given by [21-23]:

$$
\frac{d^{2} W}{d \omega d \Omega}=\left\langle\frac{d^{2} W_{e}}{d \omega d \Omega}\right\rangle\left[N_{e}+N_{e}^{2}|F(\omega, \theta)|^{2}\right]
$$

where $\left\langle\frac{d^{2} W_{e}}{d \omega d \Omega}\right\rangle$ is the weighted average of the single electron emission over the electron energy spectrum and the form factor $F(\omega, \theta)=\int \rho(\vec{x}) e^{-i \vec{k} \cdot \vec{x}} d^{3} x$ is the Fourier transform of the normalized three-dimensional bunch charge distribution $\rho(\vec{x})$, where $\vec{k}$ denotes the radiation wave vector in observation direction $\theta$ with $|\vec{k}|=\omega / c$. When the bunch size is smaller than the radiated wavelength, transition radiation emitted from individual electrons is roughly in phase, leading to a coherent enhancement represented by the second term, while the first term describes the incoherent emission. For a relativistic beam with low divergence and no correlation between transverse and longitudinal distributions, the form factor may be decomposed [23] into transverse and longitudinal components, i.e. $F=F_{\perp} F_{\|}$. If $F_{\perp}$ is known, the measured CTR spectrum directly yields the magnitude of the longitudinal form factor $F_{\|}$. Even then the longitudinal bunch profile $\rho_{\|}$cannot be found just by inverse Fourier transformation of $F_{\|}$since the phase information is not recorded.

An established reconstruction method often used in CTR experiments relies on the Kramers-Kronig relations to approximate the missing phase information [22], but this approach requires knowledge of the spectrum over the entire frequency domain. Since this is impossible, assumptions about the shape of the spectrum at low and high frequencies have to be made, and these can influence the deduced temporal profile [24,25]. In order to minimize the assumptions required, we have developed a new iterative algorithm (Bubblewrap) capable of reconstructing the longitudinal bunch profile. A detailed description of the algorithm is given in Ref. [20]. Tests with synthetic data showed accurate reconstruction results, provided that the original data covered a sufficiently broad spectral range. In the experiments reported here the necessary spectral coverage was realized by two commercial imaging spectrometers in the visible (Oriel MS260, 420-1100 nm) and nearinfrared (Princeton Instruments OMA-C with 1024element liquid nitrogen cooled InGaAs diode array, $1.1-1.8 \mu \mathrm{m}$ ) wavelength range together with a third, custom-built spectrometer sensitive up to mid-infrared wavelengths. The latter is based on a design developed at DESY-FLASH, using arrays of pyro-electric crystals $\left(\mathrm{LiTaO}_{3}\right.$, by InfraTec) to simultaneously record the intensity of the incident radiation with 60 channels in a spectral range from 1.7-7.1 $\mu \mathrm{m}$. A detailed description of a similar instrument is given in Ref. [26]. This combination of three instruments facilitates a single-shot spectral coverage from $0.4-7.1 \mu \mathrm{m}$ or $42-750 \mathrm{THz}$. The upper cutoff frequency determines the time resolution of the reconstructed bunch profiles, which amounts to $0.7 \mathrm{fs}$.

Experiments were performed using the ATLAS Ti:Sa laser system at the Max-Planck-Institut für Quantenoptik in Garching, Germany, which delivered $1.5( \pm 0.1) \mathrm{J}$ pulses of $28( \pm 2)$ fs FWHM pulse duration on target. The beam was focused by an F/22 off-axis paraboloid into a lengthtunable (3-14 mm), steady-state flow gas cell filled with hydrogen, resulting in a spot size of $18.7( \pm 1.2) \mu \mathrm{m} \mathrm{rms}$ and a normalized vector potential of $a_{0}=1.66( \pm 0.13)$. The backing pressure applied to the gas cell was controlled by a closed-loop electronic regulator with a maximum deviation of \pm 4 mbar around the target value. The reference value was taken from a calibrated pressure gauge. Due to gas flow through the laser entrance and exit holes the actual density inside the cell may be decreased. As a lower bound, we assume the gas pressure inside the cell to agree with the applied backing pressure to within 20\%. Electron bunches were accelerated following self-injection into plasma wakefields driven in the weakly relativistic regime. The experimental setup is sketched in Fig. 1. Forward CTR was generated by the passage of the relativistic electron bunch through a pair of steel tapes $(20 \mu \mathrm{m}$ thick, $25 \mathrm{~mm}$

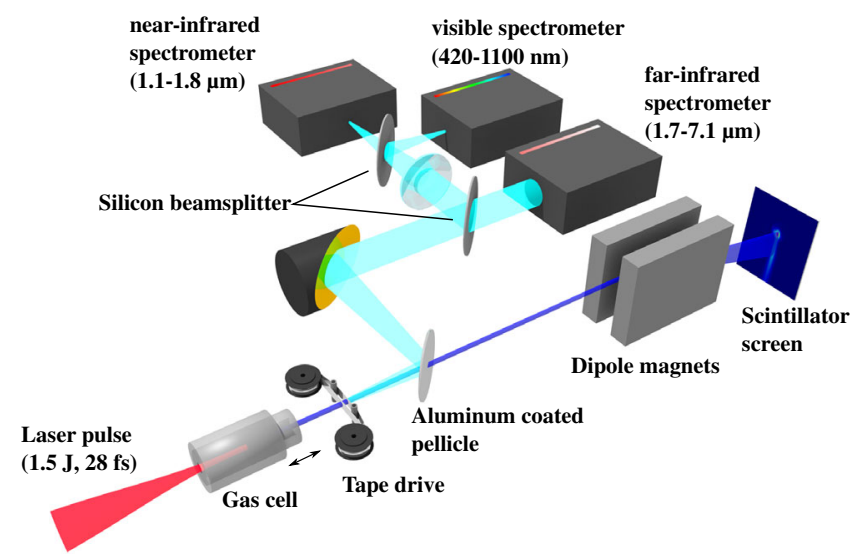

FIG. 1. Experimental setup: Accelerated electrons (dark blue) leaving the plasma generate coherent transition radiation (light blue) when they traverse the steel tapes. CTR from the 2nd tape is separated from the copropagating electron bunch, collimated by an off-axis paraboloid ( $f_{\text {eff }}=190.5 \mathrm{~mm}$ ), split by silicon wafers and directed into 3 optical spectrometers covering a spectral range from 0.4-7.1 $\mu \mathrm{m}$. The electron beam energy and charge is analyzed by a pair of dipole magnets and a scintillating screen. Additionally a second scintillating screen (not shown) located in front of the magnets can be inserted to determine the beam divergence and transverse profile. 
wide, $3 \mathrm{~mm}$ separation), located $56 \mathrm{~mm}$ behind the fixed gas cell entrance. The tapes were advanced by a motor to provide clean material for each shot. The 1st tape blocked residual laser light and ensured that no light or thermal signal could reach the back of the 2nd tape. Forward CTR produced at the backside of the 2nd tape was separated from the co-propagating electron beam by a reflective Al-coated pellicle positioned $10 \mathrm{~cm}$ behind the tape and collimated by an $f / 3.75$ off-axis paraboloid. A $1 \mathrm{~mm}$ thick silicon wafer (HRFZ-Si, by Tydex) was used as a beam splitter to reflect approximately half of the CTR radiation through a BK7 window out of the vacuum chamber. Silicon is partially transparent at wavelengths below the bandgap $(\lambda>1100 \mathrm{~nm})$, acting as a low-pass filter for the transmitted CTR signal with a flat transmission of $50( \pm 5) \%$ in the relevant spectral range from 1.7-7.1 $\mu \mathrm{m}$. The transmitted beam was directed to the mid-infrared spectrometer, which was directly connected to the main vacuum chamber. The part of the radiation reflected outside the experimental chamber was refocused by a lens $(f=60 \mathrm{~cm})$ and split by a 2nd silicon wafer of similar type onto the entrance slits of the visible and near-infrared spectrometers. The latter were absolutely calibrated, including the imaging optics, by using a $1100 \mathrm{~K}$ blackbody radiator (Lot-Oriel LSB150), a tungsten halogen lamp (Ocean Optics HL-2000-CAL) and a He-Ne laser. For the mid-infrared spectrometer, the spectral response of the instrument was calculated according to grating efficiencies (using the code MRCWA [27]) and wavelength acceptance $\Delta \lambda$ of each sensor. However, systematic variations in efficiency were found between individual pyro-elements, which typically caused the longitudinal bunch profile returned by the retrieval algorithm to contain small subsidiary bunches. These systematic variations were corrected in the following way. For experimental conditions in which only a single bunch was expected to be generated, as confirmed by the smooth shape in the visible and near-infrared spectrometers, the subsidiary electron bunches in the retrieved spectrum were filtered out, which allowed the CTR spectrum generated by the single bunch to be calculated. Comparing this with the measured spectrum then provided a correction factor for each element which could be applied in the analysis of all other data. Absolute efficiency was determined with a Nd: YAG laser. The electron beam passing straight through the pellicle was dispersed by a dipole magnet and detected by a CCD-camera on an absolutely calibrated CAWO OG16 scintillator screen [28], allowing for determination of the charge and electron energy in the range between 200$1000 \mathrm{MeV}$. A second screen could be inserted in front of the dipole magnet to monitor the transverse profile and thus the divergence after $1.5 \mathrm{~m}$ of propagation. The transverse profile was found to be approximately Gaussian and the beam divergence independent of electron energy and gas cell length. Without the radiator foil we measured a beam divergence of $1.4( \pm 0.3) \mathrm{mrad}$, yielding a transverse size of the electron bunch at the CTR radiator of $12-22 \mu \mathrm{m}$, dependent on the distance between the exit of the length-tunable gas cell and the fixed radiator foil.

The experimental setup (see Fig. 1) has the advantage that the large collection half angle of 133 mrad ensures sufficient count rates on the spectrometers compared to the smaller solid angle covered when collecting CTR behind the magnets, although one drawback is the fact that the pellicle used for separating the CTR from the electron beam is itself a source of CTR. As described in the Supplemental Material [29], the spectral transmission function of the imaging system and the interference between CTR generated at the tape and pellicle were modeled by Fourier optics propagation, taking into account the near field distribution, relative phase delay and transverse beam size at both radiators. Although they were found to be weak, interference effects were fully accounted for in our analysis. With the measured electron energy spectrum and inferred source size at both radiators, this procedure allows the CTR spectrum to be calculated in the detection plane for the case of full coherence $\left(\left|F_{\|}(\omega)\right|=1\right)$. Dividing the measured CTR spectrum by this response function then yields $\left|F_{\|}(\omega)\right|^{2}$, the square absolute value of the longitudinal form factor. The Bubblewrap retrieval algorithm is then run on this form factor to obtain the longitudinal bunch profile for each shot (see Fig. 2).

In general, the charge contained within the reconstructed bunch profile is $(80 \pm 15) \%$ of that measured independently by the electron spectrometer. Although the deviation
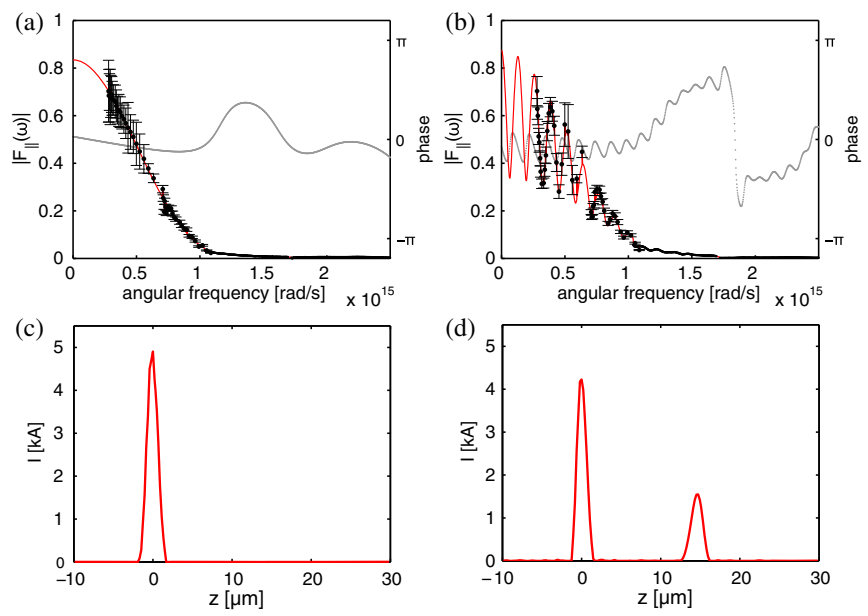

FIG. 2. Measured and reconstructed longitudinal form factor $\left|F_{\|}(\omega)\right|$ and reconstructed bunch profiles $I(z)$ for 2 representative shots for gas cell lengths of 5 and $13 \mathrm{~mm}$, respectively: (a,b) Measured (black) and reconstructed (red) CTR spectrum, and reconstructed spectral phase (grey); Error bars show the rms error of the mid-infrared spectrometer (due to readout noise, uncertainty in spectral response of individual pyro-electric detectors and transmission of the silicon wafer). (c,d) Reconstructed longitudinal bunch profiles. 

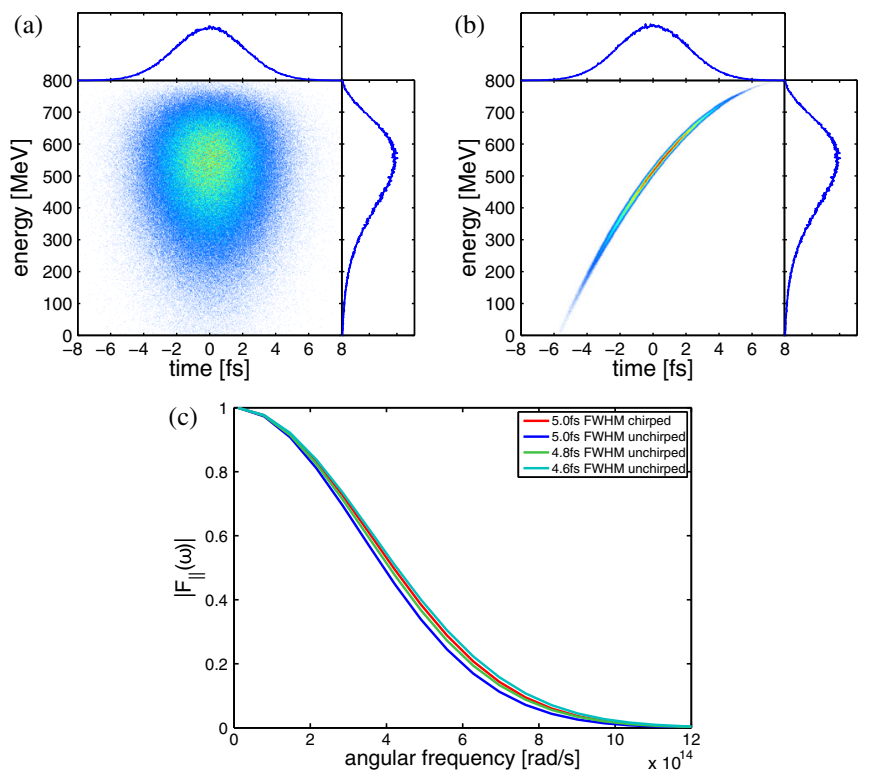

FIG. 3. Influence of a longitudinal phase-space correlation. Longitudinal phase-space distribution for (a) the case of an uncorrelated bunch and (b) for the case of a correlated bunch. (c) Simulated CTR spectra corresponding to the distributions shown in (a) and (b) for different FWHM durations.

is still within the experimental error margin, we cannot exclude a longer temporal feature consisting of low energy electrons, which would produce CTR outside of our detection range. However, such a feature could either be removed by an energy filter, or, in the view of secondary radiation sources, would contribute much less to the total emission than the high-energy part of the bunch. Furthermore, we note that our analysis does not account for a possible correlation of electron energy with longitudinal position in the electron bunch. Since the CTR emission increases with the electron energy our analysis always holds for the high-energy parts of the bunch, which are of most interest to applications. To quantify the contribution of a possible energy chirp, we have simulated CTR spectra for uncorrelated and correlated (chirped) electron beams. Figures $3(a, b)$ show the simulated longitudinal phase space distribution of an uncorrelated and a correlated beam with a Gaussian temporal shape and an identical energy spectrum. For the chirped bunch, we assumed a parabolic dependence of electron energy with longitudinal position. The computed CTR spectra for chirped and unchirped bunches are shown in Fig. 3(c). For the same FWHM duration of the longitudinal bunch profile, the chirped bunch exhibits a slightly broader CTR spectrum than an unchirped bunch. This is due to more intense CTR emitted by the high energy electrons that are concentrated in a shorter temporal feature if a chirp is present. The CTR spectrum produced by a chirped bunch of 5 fs duration is bracketed by the spectra of unchirped bunches with 4.8 fs and 4.6 fs duration (FWHM). Thus, if such a chirp were present, our method would underestimate the real bunch duration by approximately $0.3 \mathrm{fs}$.

The single-shot reconstruction technique was used in conjunction with the length tunability of the gas target to observe the evolution of the bunch profile during the acceleration process. Data were taken at $1.0 \mathrm{~mm}$ intervals for target lengths in the range $3.0-14.0 \mathrm{~mm}$ at a plasma electron density of $n_{e}=3.9 \times 10^{18} \mathrm{~cm}^{-3}$; for each target length 30 consecutive shots were recorded. For target lengths $L \leq 9.0 \mathrm{~mm}$, the CTR spectra are smooth and consequently only a single electron bunch is observed, as shown in Fig. 4(a). For a cell length optimized for maximum electron energy $(L=9.0 \mathrm{~mm})$, single electron bunches were generated with a mean cut-off energy $E_{\max }$ of $650( \pm 51) \mathrm{MeV}$, a charge of 26( \pm 6$) \mathrm{pC}$, an average bunch (a)

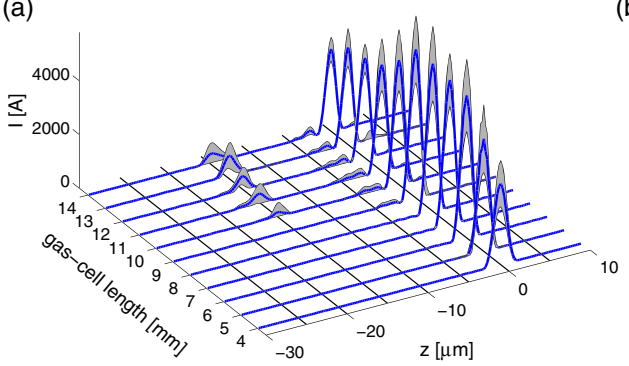

(b)

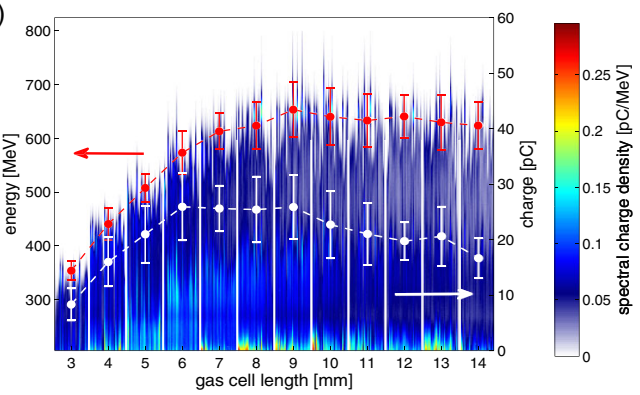

(c)

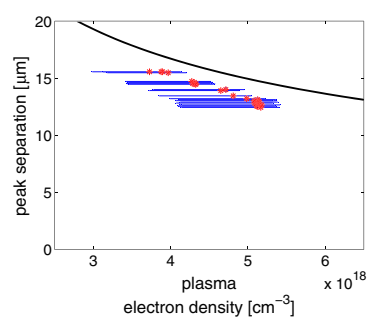

FIG. 4. (a) Bunch profile evolution at a plasma electron density of $n_{e}=3.9 \times 10^{18} \mathrm{~cm}^{-3}$ : Retrieved bunch profiles averaged over 30 consecutive shots for different gas-cell lengths. The grey band shows $\pm 1 \sigma$, where $\sigma$ is the shot-to-shot standard deviation for each 30-shot data set. (b) Electron energy evolution at $n_{e}=3.9 \times 10^{18} \mathrm{~cm}^{-3}$ : Measured electron spectra for gas cell lengths between 3 and $14 \mathrm{~mm}$, recorded in $1 \mathrm{~mm}$ steps. For each length setting, 30 consecutive electron spectra are plotted as thin vertical lines. Red dots show the average cutoff energy (set to $10 \%$ of maximum spectral charge density) at each length; white dots show the average charge. Error bars show the shot-to-shot standard deviation. (c) Measured bunch separation as a function of the plasma electron density at a fixed gas cell length of $L=12 \mathrm{~mm}$ : Error bars on the x-axis represent the uncertainty in electron density. Markers show the mean value of the bunch separation obtained in ten individual runs of the Bubblewrap algorithm, each started from a random seed. Error bars on the y-axis represent one standard deviation. The black line shows the plasma wavelength as given by 1D linear theory. 
duration of $5.1( \pm 0.2)$ fs FWHM and a peak current of $5.5( \pm 1.2) \mathrm{kA}$. A different accelerator regime was observed for target lengths greater than $9 \mathrm{~mm}$. For these conditions we observe a modulated CTR spectrum, dominated by a single frequency modulation. This modulation is characteristic for a double bunch structure, where the bunch separation $d$ is related to the modulation frequency by $\Delta \omega=2 \pi c / d$. Analysis with the Bubblewrap algorithm shows that these spectra are consistent with two distinct electron bunches separated by $15 \mu \mathrm{m}$, as shown in the plots for $L>9.0 \mathrm{~mm}$ in Fig. 4(a). Although the Bubblewrap algorithm imposes no constraints on the number of bunches a retrieved profile may contain, we always observe a double bunch structure, in agreement with the spectral modulation. In contrast, a periodic bunch train consisting of more than two bunches would exhibit a more complex CTR spectrum, containing more than one oscillation frequency. The mean charge contained in the 2nd electron bunch was observed to increase with cell length, reaching a maximum of $5 \mathrm{pC}$ at $L=13 \mathrm{~mm}$. At a fixed cell length of $L=12 \mathrm{~mm}$, we further analyzed the dependence of the bunch separation on the plasma electron density, as shown in Fig. 4(c). With increasing plasma density $n_{e}$, and thus decreasing plasma wavelength $\lambda_{p} \propto 1 / \sqrt{n_{e}}$, we also observe a decreasing bunch separation. In the tuning range of $n_{e}=3.7-5.3 \times$ $10^{18} \mathrm{~cm}^{-3}$ where stable, high-energy electron beams are observed, this separation is always found to be close to, but smaller, than $\lambda_{p}$.

Complementary information about the underlying dynamics is provided by the evolution of the electron energies [Fig. 4(b)]. Maximum energies are reached at an acceleration length of $L \approx 9.0 \mathrm{~mm}$. For $L>9.0 \mathrm{~mm}$, the maximum electron energy remains approximately constant. This indicates that the acceleration process is not limited by de-phasing, which would cause reduced electron energy with increasing target length, but by laser pump depletion and/or diffraction. For our conditions, the pump depletion length [30] $L_{p d} \approx 8.7 \frac{k_{0}^{2}}{k_{p}^{3}} \approx 10.4 \mathrm{~mm}$ is in reasonable agreement with the experimental data. For $L \geq$ $L_{p d}$ the stagnation in electron energy indicates that no laser-driven wakefield is present. In this regime, an estimate of the main bunch's charge density yields $n_{b}=$ $N_{e} /\left(2 \pi \sigma_{\perp}^{2} \int \rho_{\|}(z) d z\right) \approx 2 \times 10^{19} \mathrm{~cm}^{-3}$, where we assumed a transverse source size of $\sigma_{\perp} \approx 0.95 \mu \mathrm{m}$ (determined in our previous work with a similar experimental setup [31]). The bunch is therefore overdense with respect to the plasma density $\left(n_{b} / n_{e} \approx 5\right)$ and its transverse and longitudinal size $\left(k_{p} \sigma_{\perp} \approx 0.35, k_{p} \sigma_{\|} \approx 0.24\right)$ are small enough to drive its own wakefield in the blowout regime, according to PWFA theory [32]. With the appearance of the 2nd bunch closely linked to the point of laser energy depletion, we conjecture that a renewed injection is associated with the change from a laser driven to a particle beam driven wakefield. This injection may be assisted by a temporal slowdown of the plasma wave phase velocity $[33,34]$ as the front of the plasma wave is retarded from its position during LWFA mode toward the driving bunch. Our hypothesis is supported by the observation that: (i) for target lengths exceeding $10 \mathrm{~mm}$ the laser energy is depleted such that the bunch propagates without further (laser-induced) de- or acceleration. Due to depletion, injection of a 2 nd bunch by nonlinear wavebreaking is unlikely to occur; (ii) the measured bunch separation is smaller than the plasma wavelength $\lambda_{p}$, which indicates that both bunches reside within the same plasma cavity. Assuming the injection of a 2nd bunch would take place at the crest of a trailing plasma cavity, this separation would equal or exceed $\lambda_{p}$. We have further conducted particle-in-cell simulation with the code OSIRIS $[35,36]$ to quantify the plasma wavelength in the beam driven case (see Supplemental Material [29]). In these simulations, the expected distance between driver and witness bunch agrees with the measured value within the uncertainty related to the plasma density inside the gas cell.

In summary, we have undertaken the first measurements to elucidate the temporal dynamics of electron bunches produced by an LWFA. The broadband, high spectral resolution measurements - coupled with a novel analysis method which does not assume the shape of the spectrum in unmeasured regions-allowed us to retrieve the longitudinal bunch profile on a single-shot basis. Our results show a distinct change in the time structure of the electron beam from a single bunch to a double bunch structure. In the future, we expect that this approach will allow for a more detailed understanding about the acceleration process as well as possible changes in acceleration mode. For an acceleration length exceeding the pump depletion length the time structure consists of two separate bunches, in which one bunch is sufficiently dense to drive a wakefield in the blowout regime of PWFA. This structure is well suited for driver-witness type experiments, in particular for afterburner acceleration, where a witness bunch is accelerated in the wakefield of the first, higher charge bunch to several times the initial energy [18,37]. Our results may facilitate the study of this hybrid accelerator by avoiding the need for an externally injected bunch from a prior stage.

We would like to thank Jorge Vieira for useful discussion and support with the simulation code OSIRIS. This work was supported by the German Research Foundation (DFG) through the Cluster of Excellence Munich-Centre for Advanced Photonics (EXC 158) and SFB/TR18 funding schemes, the Euratom research and training programme 2014-2018 under Grant Agreement No. 633053 within the framework of the EUROfusion Consortium, the MaxPlanck-Society, the Engineering and Physical Sciences Research Council (Grant No. EP/H011145/1) and the Leverhulme Trust (Grant No. F/08776/G). 
[1] J. Faure, Y. Glinec, A. Pukhov, S. Kiselev, S. Gordienko, E. Lefebvre, J.-P. Rousseau, F. Burgy, and V. Malka, Nature (London) 431, 541 (2004).

[2] C. G. R. Geddes, C. Toth, J. van Tilborg, E. Esarey, C. B. Schroeder, D. Bruhwiler, C. Nieter, J. Cary, and W. P. Leemans, Nature (London) 431, 538 (2004).

[3] S. P. D. Mangles, C. D. Murphy, Z. Najmudin, A. G. R. Thomas, J. L. Collier, A. E. Dangor, E. J. Divall, P. S. Foster, J. G. Gallacher, C. J. Hooker, D. A. Jaroszynski, A. J. Langley, W. B. Mori, P. A. Norreys, F. S. Tsung, R. Viskup, B. R. Walton, and K. Krushelnick, Nature (London) 431, 535 (2004).

[4] W. P. Leemans, B. Nagler, A. J. Gonsalves, C. Tth, K. Nakamura, C. G. R. Geddes, E. Esarey, C. B. Schroeder, and S. M. Hooker, Nat. Phys. 2, 696 (2006).

[5] J. Osterhoff, A. Popp, Z. Major, B. Marx, T. P. RowlandsRees, M. Fuchs, M. Geissler, R. Hörlein, B. Hidding, S. Becker, E. A. Peralta, U. Schramm, F. Grüner, D. Habs, F. Krausz, S. M. Hooker, and S. Karsch, Phys. Rev. Lett. 101, 085002 (2008), 1.

[6] T. Seggebrock, A. R. Maier, I. Dornmair, and F. Grüner, Phys. Rev. ST Accel. Beams 16, 070703 (2013).

[7] F. Grüner, S. Becker, U. Schramm, T. Eichner, M. Fuchs, R. Weingartner, D. Habs, J. Meyer-ter Vehn, M. Geissler, M. Ferrario, L. Serafini, B. v. d. Geer, H. Backe, W. Lauth, and S. Reiche, Appl. Phys. B 86, 431 (2007).

[8] N. D. Powers, I. Ghebregziabher, G. Golovin, C. Liu, S. Chen, S. Banerjee, J. Zhang, and D. P. Umstadter, Nat. Photonics 8, 28 (2014).

[9] A. Rousse, K. T. Phuoc, R. Shah, A. Pukhov, E. Lefebvre, V. Malka, S. Kiselev, F. Burgy, J.-P. Rousseau, D. Umstadter, and D. Hulin, Phys. Rev. Lett. 93, 135005 (2004).

[10] J. Wenz, S. Schleede, K. Khrennikov, M. Bech, P. Thibault, M. Heigoldt, F. Pfeiffer, and S. Karsch, Nat. Commun. 6, 7568 (2015).

[11] K. Khrennikov, J. Wenz, A. Buck, J. Xu, M. Heigoldt, L. Veisz, and S. Karsch, Phys. Rev. Lett. 114, 195003 (2015).

[12] A. Buck, M. Nicolai, K. Schmid, C. M. S. Sears, A. Savert, J. M. Mikhailova, F. Krausz, M. C. Kaluza, and L. Veisz, Nat. Phys. 7, 543 (2011).

[13] O. Lundh, J. Lim, C. Rechatin, L. Ammoura, A. Ben-Ismail, X. Davoine, G. Gallot, J.-P. Goddet, E. Lefebvre, V. Malka, and J. Faure, Nat. Phys. 7, 219 (2011).

[14] O. Lundh, C. Rechatin, J. Lim, V. Malka, and J. Faure, Phys. Rev. Lett. 110, 065005 (2013).

[15] E. Esarey, C. B. Schroeder, and W. P. Leemans, Rev. Mod. Phys. 81, 1229 (2009).

[16] I. Blumenfeld, C. E. Clayton, F.-J. Decker, M. J. Hogan, C. Huang, R. Ischebeck, R. Iverson, C. Joshi, T. Katsouleas, N. Kirby, W. Lu, K. A. Marsh, W. B. Mori, P. Muggli, E. Oz, R. H. Siemann, D. Walz, and M. Zhou, Nature (London) 445, 741 (2007).

[17] P. Muggli, B. E. Blue, C. E. Clayton, S. Deng, F.-J. Decker, M. J. Hogan, C. Huang, R. Iverson, C. Joshi, T. C. Katsouleas, S. Lee, W. Lu, K. A. Marsh, W. B. Mori, C. L. O'Connell, P. Raimondi, R. Siemann, and D. Walz, Phys. Rev. Lett. 93, 014802 (2004).
[18] P. Chen, J. M. Dawson, R. W. Huff, and T. Katsouleas, Phys. Rev. Lett. 54, 693 (1985).

[19] M. J. Hogan, T. O. Raubenheimer, A. Seryi, P. Muggli, T. Katsouleas, C. Huang, W. Lu, W. An, K. A. Marsh, W. B. Mori, C. E. Clayton, and C. Joshi, New J. Phys. 12, 055030 (2010).

[20] S. I. Bajlekov, M. Heigoldt, A. Popp, J. Wenz, K. Khrennikov, S. Karsch, and S. M. Hooker, Phys. Rev. ST Accel. Beams 16, 040701 (2013).

[21] C. J. Hirschmugl, M. Sagurton, and G. P. Williams, Phys. Rev. A 44, 1316 (1991).

[22] R. Lai and A. Sievers, Nucl. Instrum. Methods Phys. Res., Sect. A 397, 221 (1997).

[23] C. B. Schroeder, E. Esarey, J. van Tilborg, and W. P. Leemans, Phys. Rev. E 69, 016501 (2004).

[24] D. Mihalcea, C. L. Bohn, U. Happek, and P. Piot, Phys. Rev. ST Accel. Beams 9, 082801 (2006).

[25] D. Pelliccia and T. Sen, Nucl. Instrum. Methods Phys. Res., Sect. A 764, 206 (2014).

[26] S. Wesch, B. Schmidt, C. Behrens, H. Delsim-Hashemi, and P. Schmüser, Nucl. Instrum. Methods Phys. Res., Sect. A 665, 40 (2011).

[27] M. G. Moharam, D. A. Pommet, E. B. Grann, and T. K. Gaylord, J. Opt. Soc. Am. A 12, 1077 (1995).

[28] A. Buck, K. Zeil, A. Popp, K. Schmid, A. Jochmann, S. D. Kraft, B. Hidding, T. Kudyakov, C. M. S. Sears, L. Veisz, S. Karsch, J. Pawelke, R. Sauerbrey, T. Cowan, F. Krausz, and U. Schramm, Rev. Sci. Instrum. 81, 033301 (2010).

[29] See Supplemental Material at http://link.aps.org/ supplemental/10.1103/PhysRevSTAB.18.121302 for a detailed description of the spectrometer response function and a description of PIC simulations that quantify the expected distance between driver and witness bunch in the beam driven case.

[30] B. A. Shadwick, C. B. Schroeder, and E. Esarey, Phys. Plasmas 16, 056704 (2009).

[31] R. Weingartner, S. Raith, A. Popp, S. Chou, J. Wenz, K. Khrennikov, M. Heigoldt, A. R. Maier, N. Kajumba, M. Fuchs, B. Zeitler, F. Krausz, S. Karsch, and F. Grner, Phys. Rev. ST Accel. Beams 15, 111302 (2012).

[32] J. B. Rosenzweig, B. Breizman, T. Katsouleas, and J. J. Su, Phys. Rev. A 44, R6189 (1991).

[33] S. Bulanov, N. Naumova, F. Pegoraro, and J. Sakai, Phys. Rev. E 58, R5257 (1998).

[34] P. Tomassini, M. Galimberti, A. Giulietti, D. Giulietti, L. A. Gizzi, L. Labate, and F. Pegoraro, Phys. Rev. ST Accel. Beams 6, 121301 (2003).

[35] R. Fonseca, L. Silva, F. Tsung, V. Decyk, W. Lu, C. Ren, W. Mori, S. Deng, S. Lee, T. Katsouleas, and J. Adam, in Computational Science ICCS 2002, Lecture Notes in Computer Science, edited by P. Sloot, A. Hoekstra, C. Tan, and J. Dongarra (Springer, Berlin, Heidelberg, 2002), Vol. 2331, p. 342.

[36] R. A. Fonseca, J. Vieira, F. Fiuza, A. Davidson, F. S. Tsung, W. B. Mori, and L. O. Silva, Plasma Phys. Controlled Fusion 55, 124011 (2013).

[37] B. Hidding, T. Königstein, J. Osterholz, S. Karsch, O. Willi, and G. Pretzler, Phys. Rev. Lett. 104, 195002 (2010). 